\title{
Helicity-vorticity turbulent pumping of magnetic fields in the solar dynamo
}

\author{
V. V. Pipin \\ Institute Solar-Terrestrial Physics, Irkutsk, Russia \\ email:pip@iszf.irk.ru
}

\begin{abstract}
The interaction of helical convective motions and differential rotation in the solar convection zone results in turbulent drift of a large-scale magnetic field. We discuss the pumping mechanism and its impact on the solar dynamo.
\end{abstract}

Keywords. Turbulence; Mean-field magnetohydrodynamics; Sun; magnetic field; Stars: Dynamo activity

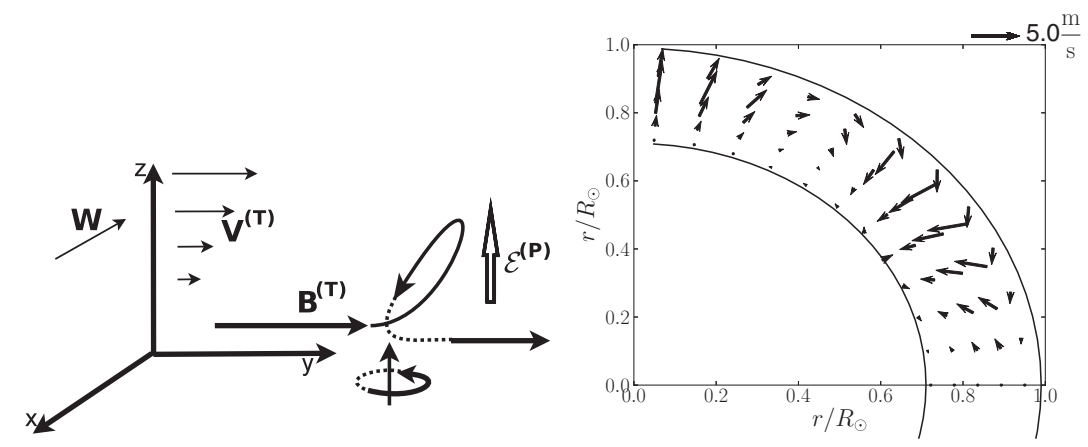

Figure 1. The field lines of the large-scale magnetic field, $\boldsymbol{B}^{(T)}$, are transformed by the helical motions to a twisted $\Omega$-like shape. This loop is folded by the large-scale shear, $\boldsymbol{V}^{(T)}$, into the direction of the background large-scale magnetic field, $\boldsymbol{B}^{(T)}$. The induced electromotive force has a component, $\mathcal{E}^{(P)}$, which is perpendicular to the field $\boldsymbol{B}^{(T)}$. The resulting effect is identical to the effective drift of the large-scale magnetic field along the $x$-axis, in the direction opposite to the large-scale vorticity vector $\boldsymbol{W}=\boldsymbol{\nabla} \times \boldsymbol{V}^{(T)}$, i.e., $\mathcal{E}^{(P)} \sim-\boldsymbol{W} \times \boldsymbol{B}^{(T)}$.

\section{Introduction}

Recently (Pipin 2008; Mitra et al. 2009; Leprovost \& Kim 2010), it has been found that the helical convective motions and the helical turbulent magnetic fields interacting with large-scale magnetic fields and differential rotation can produce effective pumping in the direction of the large-scale vorticity vector. Figure 1 illustrates the principal processes that induce the helicity-vorticity pumping effect. A comprehensive study of the linear helicity-vorticity pumping effect for the case of weak shear and slow rotation was given by Rogachevskii et al. (2011) and their results were extended by the direct numerical simulations (DNS) with a more general test-field method Brandenburg et al. (2012). 
a)

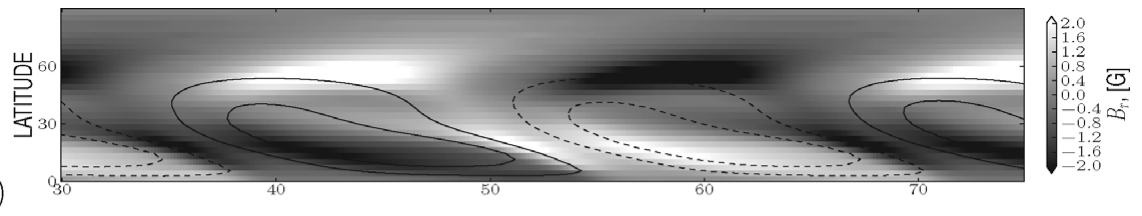

b)

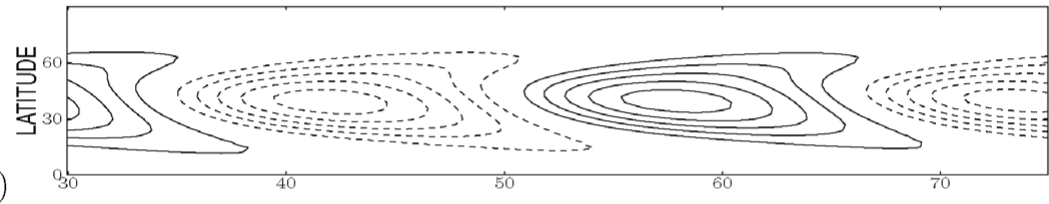

c)

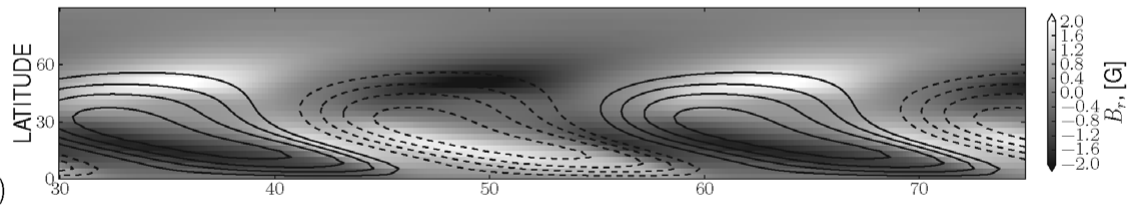

d)

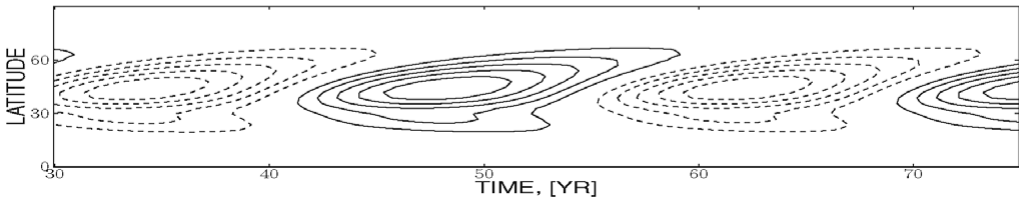

Figure 2. The time-latitude diagrams for the toroidal and radial magnetic fields for the models $\mathrm{D} 1$ and D2: a) the model D1, the toroidal field (iso-contours, $\pm .25 K G$ ) near the surface and the radial field (gray-scale density plot); b) the model D1, the toroidal field at the bottom of the solar convection zone, the contours drawn in the range $\pm .5 \mathrm{KG}$; c) the same as for item a) for the model D2; d) the same as for item b) for the model D2.

\section{The solar dynamo model}

It is found that the magnetic helicity contribution of the pumping effect can be important for explaining the fine structure of the sunspot butterfly diagram. In particular, the magnetic helicity contribution results in a slow-down of equatorial propagation of the dynamo wave. The slow-down starts just before the maximum of the cycle. For the time being it is unclear what are the differences in predictions between different dynamo models and how well do they reproduce the observations. A more detailed analysis is needed.

Acknowledgments. I thank for the support the RFBR grants 12-02-00170-a, 10-0200148-a and 10-02-00960, the support of the Integration Project of SB RAS N 34, and support of the state contracts 02.740.11.0576, 16.518.11.7065 of the Ministry of Education and Science of Russian Federation.

\section{References}

Brandenburg, A., Rädler, K.-H., \& Kemel, K. 2012, Astron. Astrophys., 539, A35

Leprovost, N. \& Kim, E.-J. 2010, Geophysical and Astrophysical Fluid Dynamics, 104, 167

Mitra, D., Käpylä, P. J., Tavakol, R., \& Brandenburg, A. 2009, Astron. Astrophys., 495, 1

Pipin, V. V. 2008, Geophysical and Astrophysical Fluid Dynamics, 102, 21

Rogachevskii, I., Kleeorin, N., Käpylä, P. J., \& Brandenburg, A. 2011, Phys. Rev. E, 84, 056314 